\title{
GENETIC ASPECTS OF FAMILIAL AMYLOID POLYNEUROPATHY IN OGAWA VILLAGE, JAPAN
}

\author{
Eiko Itoga and Shozo KITo \\ Third Department of Internal Medicine, Hiroshima University \\ School of Medicine, Hiroshima 734, Japan
}

Summary Genetic studies were made in 120 males and 92 females of 408 constituents of 22 pedigrees of familial amyloid polyneuropathy (FAP) originated from Ogawa Village area, Japan. The results were as follows.

1) The ages at the onset ranged from 20 to 68 , and the mean age was 35.38 in males and 37.88 in females.

2) The cumulative morbid risk ratio was high as 0.8 and above.

3) The penetrance was very high, i.e. $100 \%$ when calculated from parents' side and $99.29 \%$ from children's side. No skipping of generations was noticed except a case in which the determination of "affected" or "non affected" was impossible.

4) The segregation ratios were $34.67 \pm 27.50 \%$ as calculated by a single selection and $45.80 \pm 2.86 \%$ by a complete selection.

5) The transmission mode of FAP originated from Ogawa Village, Japan was autosomaly dominant.

The highness of the cumulative morbid risk ratio and penetrance ratio suggests high incidence of this disease, in future too, and the early etiological clarification is desired.

\section{INTRODUCTION}

Familial amyloid polyneuropathy (FAP) was established as a clinical entity by Andrade (1952). Its one of major signs is polyneuropathy and amyloid substance accumulates characteristically in peripheral nerves as well as in other various organs.

As for Japanese FAP cases, Araki et al. (1968) reported for the first time, cases from Arao city in Kyushu Island, Japan. Kito et al. (1973) discovered the second largest conglomeration of the disease of the world in the Central Japan. The region was a remote and isolated mountain village named, Ogawa which had been notorious for so-called "leprosy" for hundreds of years. Figure 1 shows a FAP patient in this area. Various reports have been also made on FAP in this area by a number of other 


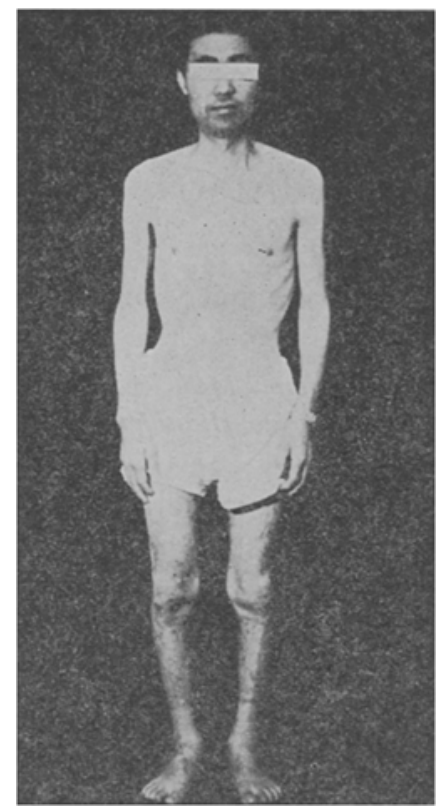

Fig. 1. The full-length photograph of a FAP patient in Ogawa Village.

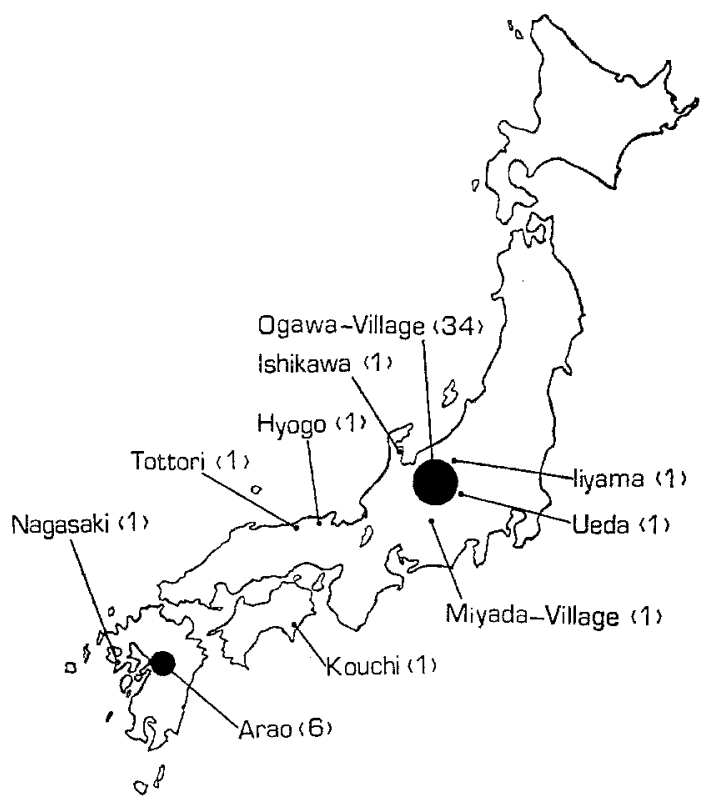

Fig. 2. Geographic distribution of FAP in Japan. The numbers of families are shown in parentheses.

Jpn. J. Human Genet. 
Table 1. Signs and symptoms in FAP cases of the Ogawa Village area (Kito et al., 1980).

\begin{tabular}{lrlr}
\hline Superficial sensory dullness with & & Orthostatic hypotension & 47 \\
dissociation & 65 & Nausea or vomiting & 42 \\
Constipation & 63 & Menstrual abnormalities & $9 / 36$ \\
Diarrhoea & 61 & Trophic disturbance of the skin & 40 \\
Impotence & $31 / 46$ & Spontaneous pain & 35 \\
Muscle atrophy with weakness & 52 & Dyshydrosis & 22 \\
Pupillary deformities & 55 & Macroglossia & 23 \\
Dysuria & 56 & Glaucoma & 11 \\
Loss of body weight & 48 & Skin edema & 3 \\
Absence of light reflex & 44 & Hepatomegaly & 3 \\
Anisocoria & 43 & Struma & 13 \\
Insular area of sensory dullness & & Charcot's joint & 5 \\
$\quad$ in the front chest & 37 & Opacities of the vitreous & 2 \\
\hline
\end{tabular}

authors (Nakao et al., 1966; Yamazaki et al., 1969; Izawa, et al., 1969; Satoyoshi et al., 1971; Takaya et al., 1972; Tsubura et al., 1973 and Oda et al., 1974). At present, the geographic distribution of FAP in Japan is as shown in Fig. 2.

Kito et al. investigated HLA-disease association with FAP in the Ogawa Village area in 1976, and reported the signs and symptoms of the disease in this area (Table 1) and the result of dimethyl sulfoxide (DMSO) treatment in 1980. The symptomatological features of each pedigree of this disease and its high incidence in this area stress the necessity of a genetic investigation.

In this study, human genetical features of the pedigrees in the Ogawa Village area will be presented on the basis of the following epidemiological research.

\section{MATERIALS AND METHODS}

Materials are the cases of FAP originating from the Ogawa Village area, Japan. Four hundreds and eight members of 22 pedigrees in Ogawa Village area were studied in this genetical investigation.

We obtained informations on the members whom we could not examine directly at medical facilities through various ways. As for those who had died, we estimated whether they were FAP patients or not by surveys of their histories and hospital protocols. Precise clinical and laboratory examinations were performed on 82 cases from the Ogawa Village area. The details of these results were described in the previous paper (Kito et al., 1980). The diagnosis of amyloidosis was made by sural nerve biopsies in these cases.

The genetic calculations were made by the methods of Tanaka (1978), Fisher (Maximum-Likelihood-Method) (Ohkura, 1979) and Matsunaga (1979). Segregation ratios were corrected by the penetrance parameter due to delayed onset (Kondo, 
1976 and Morton, 1959). We regarded the suspected cases as FAP ones and the cases on which diagnosis was impossible as non.FAP ones in each calculation. But for the calculations of penetrance ratios, the unconfirmed cases are excluded. We also excluded the young people (under the age of 20) in whom either signs or symptoms of FAP were not yet developed in a final confirmation.

\section{RESULTS}

In this study, the population of the cases originating from Ogawa Village consisted of 22 pedigrees, 408 constituents and 108 sibship groups and affected sibs were 120 males and 92 females (Table 2).

Table 2. Number of affected sibships.

\begin{tabular}{|c|c|c|c|c|c|c|c|c|}
\hline & \multicolumn{3}{|c|}{ Affected sibs } & \multirow{2}{*}{$\begin{array}{l}\text { Normal } \\
\text { sibs }\end{array}$} & \multicolumn{2}{|c|}{ (Unknown) } & \multirow{2}{*}{$\begin{array}{l}\text { Total } \\
\text { sibs }\end{array}$} & \multirow{2}{*}{$\begin{array}{l}\text { Number } \\
\text { of sibships }\end{array}$} \\
\hline & $\mathrm{m}$ & $\mathrm{f}$ & Total & & $\mathrm{m}$ & f & & \\
\hline $\mathrm{r}_{\mathrm{min}}=1$ & 120 & 92 & 212 & 196 & 4 & 2 & 408 & 108 \\
\hline$I_{\min }=2$ & 100 & 81 & 181 & 116 & 4 & 2 & 297 & 67 \\
\hline
\end{tabular}

$\mathrm{r}_{\min }=1$ : at least one affected individual. $\quad \mathrm{r}_{\min }=2:$ at least two affected individuals.
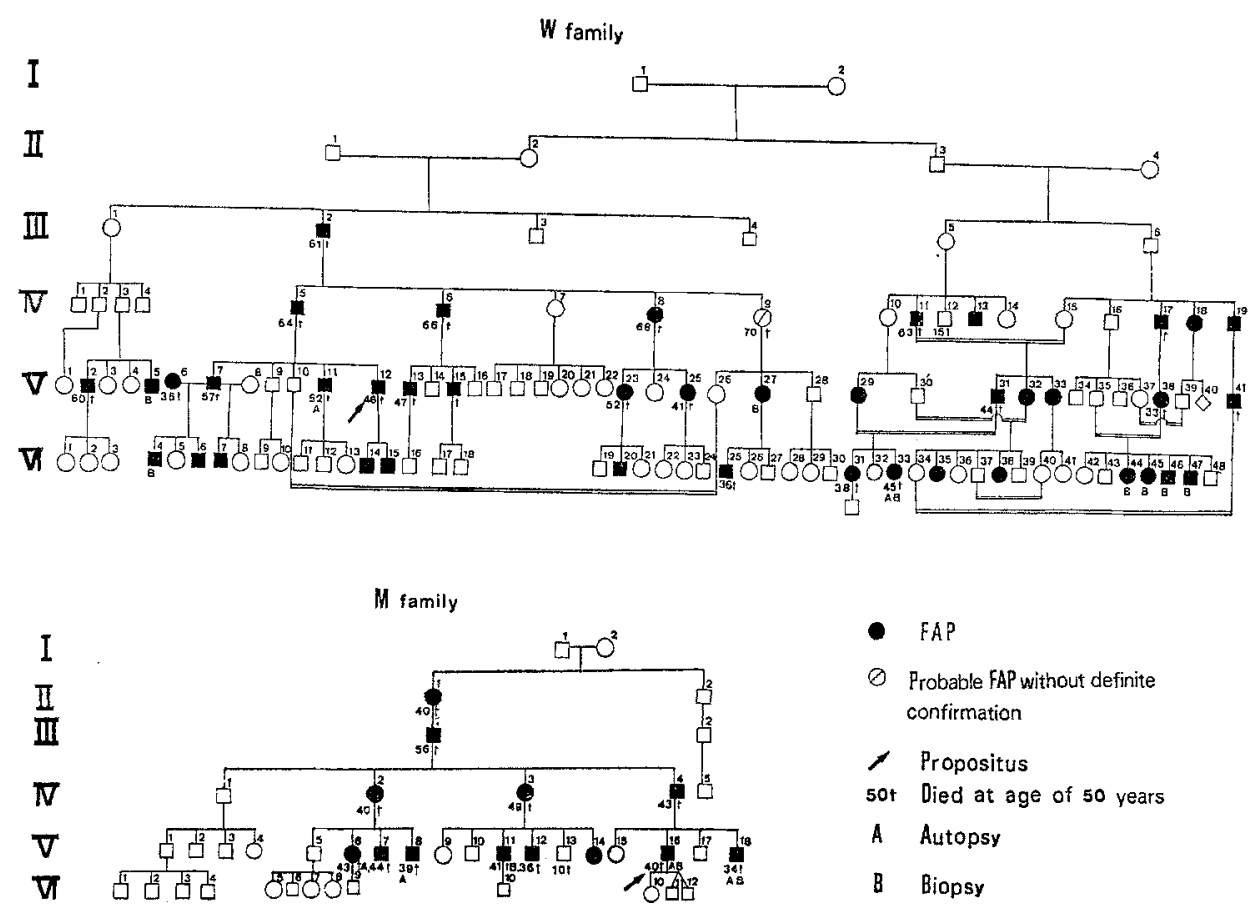

Fig. 3. Examples of the pedigree maps of FAP in Ogawa Village. 

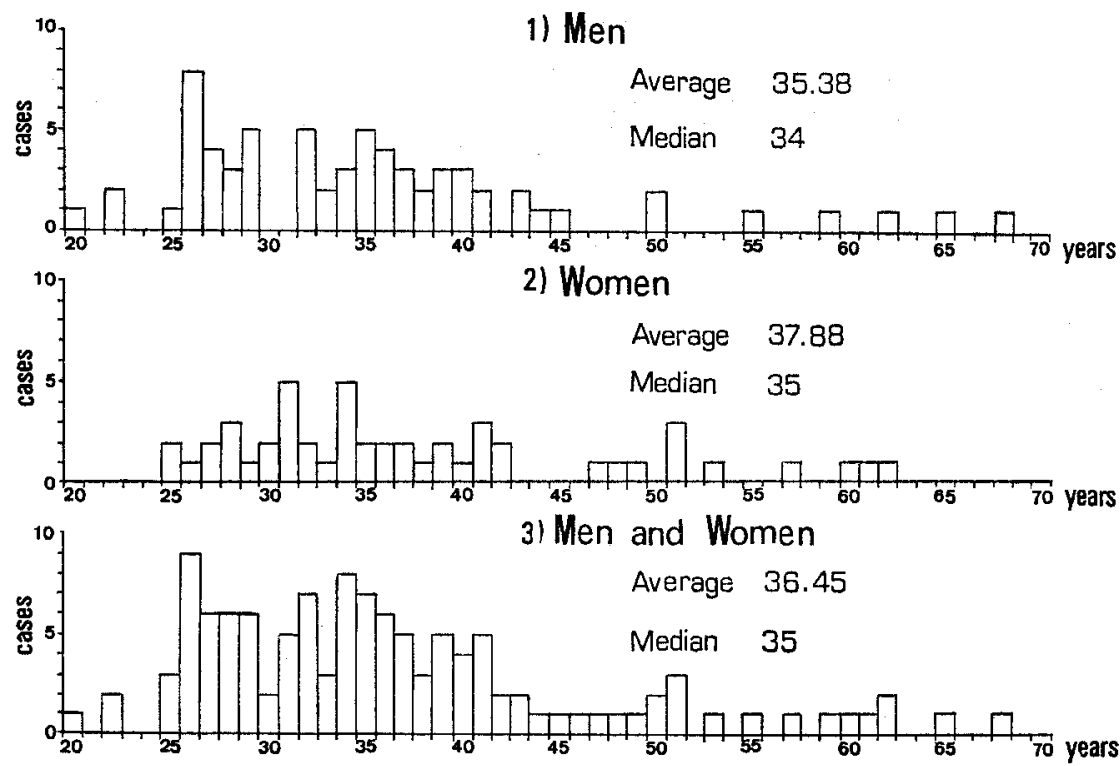

Fig. 4. Distribution of the onset age of FAP in Ogawa Village.

Figure 3 shows some examples of the pedigree maps of FAP originating from the Ogawa Village area. These maps indicate that FAP is transmitted not only from the same sex but also directly from male to female and from female to male.

The ages at the onset of 117 patients (67 males and 50 females) on whom FAP was definitely diagnosed ranged between 20 and 68 years. The mean age was $\mathbf{3 5 . 3 8}$ in male and 37.88 in female. The median age was 34 in male and $\mathbf{3 5}$ in female (Fig. 4).

We calculated genetic penetrance ratios according to the following expressions (Tanaka, 1978).

The penetrance ratio from parents' side;

$$
\mathrm{P}=\frac{\mathrm{a}}{\mathrm{b}} \times 100
$$

a: Number of the families in which at least one parent has the trait.

b: Number of the families in which children have the trait.

The penetrance ratio from children's side;

$$
P=\frac{2 a}{a+n} \times 100
$$

The families in which a parent is a heterozygote and the other parent is a normal homozygote are selected.

a: Number of affected children.

$\mathrm{n}$ : Number of non-affected children. 
All the penetrance ratios from parents' side were $100 \%$ and the skipping of generations was not observed except a case in which the determination of "affected" of "non-affected" was impossible. The penetrance ratios from children's side were between $28.57 \%$ and $200 \%$ and differed with families. The penetrance ratio from children's side was $99.29 \%$ in all the families (Table 3).

The segregation ratios were calculated on the basis shown in Table 4. Calculating the segregation ratio were performed by the two means of ascertaining affected individuals: complete ascertainment (selection) which ascertained all affected sibs in a community and incomplete single ascertainment (selection) which ascertained each affected family through its only one affected sibs no matter how many affected there might be. The calculations of the segregation ratios were based on the next expressions (Ohkura, 1979).

Table 3. Penetrance ratio.

\begin{tabular}{|c|c|c|c|c|c|c|c|}
\hline \multirow{2}{*}{$\begin{array}{l}\text { No. of } \\
\text { families }\end{array}$} & \multicolumn{2}{|c|}{$\begin{array}{l}\text { No. of } \\
\text { parent's sex }\end{array}$} & \multirow{2}{*}{$\begin{array}{l}\text { No. of } \\
\text { patients }\end{array}$} & \multirow{2}{*}{$\begin{array}{l}\text { No. of } \\
\text { normal } \\
\text { members }\end{array}$} & \multirow{2}{*}{$\begin{array}{c}\text { No. of } \\
\text { normal } \\
\text { parents of } \\
\text { patients }\end{array}$} & \multicolumn{2}{|c|}{ Penetrance ratio } \\
\hline & male & female & & & & $\begin{array}{l}\text { from } \\
\text { children's side }\end{array}$ & $\begin{array}{l}\text { from } \\
\text { parents' side }\end{array}$ \\
\hline 1 & 2 & 2 & 11 & 6 & 0 & $129.41 \%$ & $100 \%$ \\
\hline 2 & 0 & 1 & 1 & 3 & 0 & 50 & 100 \\
\hline 3 & 1 & 2 & 5 & 3 & 0 & 125 & 100 \\
\hline 5 & 3 & 0 & 5 & 5 & 0 & 100 & 100 \\
\hline 6 & 1 & 1 & 3 & 5 & 0 & 75 & 100 \\
\hline 7 & 1 & 1 & 5 & 7 & 0 & 83.33 & 100 \\
\hline 8 & 4 & 3 & 12 & 7 & 0 & 126.32 & 100 \\
\hline 9 & 1 & 4 & 11 & 8 & 0 & 115.79 & 100 \\
\hline 10 & 2 & 0 & 4 & 3 & 0 & 114.29 & 100 \\
\hline 11 & 1 & 1 & 8 & 5 & 0 & 123.08 & 100 \\
\hline 12 & 0 & 2 & 2 & 4 & 0 & 66.67 & 100 \\
\hline 13 & 6 & 4 & 15 & 16 & 0 & 96.77 & 100 \\
\hline 14 & 2 & 1 & 6 & 4 & 0 & 120 & 100 \\
\hline 15 & 2 & 5 & 17 & 12 & 0 & 117.24 & 100 \\
\hline 16 & 1 & 1 & 2 & 6 & 0 & 50 & 100 \\
\hline 17 & 1 & 1 & 4 & 7 & 0 & 72.73 & 100 \\
\hline 18 & 2 & 3 & 9 & 16 & 0 & 72 & 100 \\
\hline 19 & 8 & 5 & 24 & 22 & 0 & 104.34 & 100 \\
\hline 21 & 0 & 1 & 1 & 0 & 0 & 200 & 100 \\
\hline 22 & 0 & 1 & 1 & 6 & 0 & 28.57 & 100 \\
\hline Total & 38 & 39 & 139 & 141 & 0 & 99.29 & 100 \\
\hline
\end{tabular}


Table 4. Classification of the sibships according to number of affected individuals and size of sibships.

\begin{tabular}{|c|c|c|c|c|c|c|c|c|c|c|c|c|}
\hline & $s=$ & 1 & 2 & 3 & 4 & 5 & 6 & 7 & 8 & 9 & $\sum_{\mathbf{s} \geqq 1}(\cdots)$ & $\sum_{\mathbf{s} \geqq 2}(\cdots)$ \\
\hline \multirow{5}{*}{$\mathrm{r}=$} & 1 & 12 & 9 & 9 & 6 & 2 & 1 & 2 & & & 41 & 29 \\
\hline & 2 & & 4 & 15 & 15 & 4 & 2 & 1 & & & 41 & 41 \\
\hline & 3 & & & 2 & 4 & 6 & 2 & 1 & & 1 & 16 & 16 \\
\hline & 4 & & & & & 3 & 2 & 1 & 1 & 2 & 9 & 9 \\
\hline & 5 & & & & & 1 & & & & & 1 & 1 \\
\hline \multirow{3}{*}{$T \geqq 1$} & $\sum_{\mathrm{r}} \mathrm{n}_{\mathrm{sr}}$ & 12 & 13 & 26 & 25 & 16 & 7 & 5 & 1 & 3 & 108 & 96 \\
\hline & $\sum \mathrm{rn}_{\mathrm{Sr}}$ & 12 & 17 & 45 & 48 & 45 & 19 & 11 & 4 & 11 & 212 & 200 \\
\hline & $\sum_{\mathrm{r}} \mathrm{sn}_{\mathrm{sr}}$ & 12 & 26 & 78 & 100 & 80 & 42 & 35 & 8 & 27 & 408 & 396 \\
\hline \multirow{3}{*}{$r \geqq 2$} & $\sum_{\mathrm{r}} \mathrm{n}_{\mathrm{sr}}$ & & 4 & 17 & 19 & 14 & 6 & 3 & 1 & 3 & & 67 \\
\hline & $\sum_{\mathrm{r}} \mathrm{rn}_{\mathrm{Br}}$ & & 18 & 36 & 42 & 43 & 18 & 9 & 4 & 11 & & 181 \\
\hline & $\sum_{\mathrm{r}} \mathrm{Sn}_{\mathrm{sr}}$ & & 8 & 51 & 76 & 70 & 36 & 21 & 8 & 27 & & 297 \\
\hline
\end{tabular}

$\mathbf{s}=$ size of sibships, $\mathbf{r}=$ affected sibs.

$\mathrm{n}_{\mathrm{sr}}=$ sibships of size $\mathrm{s}$ comprising $\mathrm{r}$ affected sibs in sibships involving at least one, or at least two affected sibs.

The method for calculating the segregation ratio;

Single selection;

$$
\mathrm{P}=\frac{\mathrm{R}-\mathrm{N}}{\mathrm{T}-\mathrm{N}}, \quad \mathrm{Vp}=\frac{(\mathrm{T}-\mathrm{R})(\mathrm{R}-\mathrm{N})}{(\mathrm{T}-\mathrm{N}) 3}
$$

T: Number of sibs.

N: Number of sibship groups.

R: Number of affected sibs in all sibship groups.

Complete selection (Maximum-Likelihood-Method);

$$
\frac{\mathrm{R}}{\mathrm{P}}=\Sigma \frac{\mathrm{SNsr}}{1-\mathrm{qs}}, \quad \mathrm{Vp}=\Sigma \frac{\mathrm{pq}(1-\mathrm{qs}) 2}{\mathrm{SNsr}(1-\mathrm{qs}-\mathrm{spq} \mathrm{s}-1)}
$$

R: Number of affected sibs in all sibship group.

S: Size of sibship group.

Nsr: Number of sibship groups of s constituents comprising $r$ affected sibs.

In the sibship group with at least one affected sib (when $r_{\min }=1$ ), the segregation ratio were calculated from Table 4 as follows.

If a single selection $(\mathrm{k}=0)$ was assumed, the corrected figure of the affected individual in the sibship was

$$
P=100 \frac{212-108}{408-108}=34.67 \pm 2.75 \%
$$


according to the expression (3). If a complete selection $(\mathrm{k}=1)$ was assumed, the corrected value was $45.80 \pm 2.86 \%$ according to the expressions (4) (Table 5).

When the way of ascertainment is not clear, the approximation of the segregation ratio lies between the value calculated with assumption of a single selection $(\mathrm{k}=0)$ and the one with assumption of a complete selection $(\mathrm{k}=1)$. Since we were apt to obtain the smallest value in a single selection and the largest one in a complete selection respectively, we assumed that the segregation ratio in $\mathrm{r}_{\min }=1$ lay between $34.67-2.75 \%$ and $45.80+2.86 \%$.

When we considered only sibship group of minimum size with 2 sibs and at

Table 5. Complete selection (Maximum-likelihood method).

\begin{tabular}{ccccccc}
\hline $\mathrm{S}$ & Nsr & Observed & $\frac{\mathrm{SP}}{1-\mathrm{q}^{\mathrm{s}}}$ & $\begin{array}{c}\mathrm{P}=0.45 \\
\text { Expected }\end{array}$ & $\frac{\mathrm{SP}}{1-\mathrm{q}^{\mathrm{s}}}$ & $\begin{array}{c}\mathrm{P}=0.475 \\
\text { Expected }\end{array}$ \\
\hline 2 & 13 & 17 & 1.290 & 16.770 & 1.311 & 17.043 \\
3 & 26 & 45 & 1.619 & 42.094 & 1.666 & 43.316 \\
4 & 25 & 48 & 1.981 & 49.525 & 2.056 & 51.400 \\
5 & 16 & 45 & 2.369 & 37.904 & 2.474 & 39.584 \\
6 & 7 & 19 & 2.777 & 19.439 & 2.911 & 20.377 \\
7 & 5 & 11 & 3.199 & 15.995 & 3.362 & 16.810 \\
8 & 1 & 4 & 3.630 & 3.630 & 3.822 & 3.822 \\
9 & 3 & 11 & 4.069 & 12.207 & 4.288 & 12.864 \\
\hline Total & 96 & 200 & & 197.564 & & 205.216
\end{tabular}

$\mathrm{P}=0.45, \mathrm{P}=0.475, \mathrm{R}=197.564, \mathrm{R}=205.216$.

Corrected proportion of affected, after linear interpolation, $P=0.4580(R=200)$.

\begin{tabular}{cccccc}
\hline $\mathrm{S}$ & $\mathrm{Nsr}$ & $\frac{\mathrm{S}\left(1-\mathrm{q}^{\mathrm{s}}-\mathrm{SPq}^{\mathrm{s}-1}\right)}{\mathrm{Pq}\left(1-\mathrm{q}^{\mathrm{s}}\right)^{2}}$ & $\mathrm{P}=0.45$ & $\frac{\mathrm{S}\left(1-\mathrm{q}^{\mathrm{s}}-\mathrm{SP}-\mathrm{q}^{\mathrm{s}-1}\right)}{\mathrm{Pq}\left(1-\mathrm{q}^{\mathrm{s}}\right)^{2}}$ & $\begin{array}{c}\mathrm{P}=0.475 \\
\mathrm{~W}\end{array}$ \\
\hline 2 & 13 & 3.364 & 43.732 & 3.499 & 44.837 \\
3 & 26 & 7.417 & 192.842 & 7.606 & 197.756 \\
4 & 25 & 11.925 & 298.125 & 12.194 & 304.850 \\
5 & 16 & 16.661 & 266.576 & 16.959 & 271.344 \\
6 & 7 & 21.448 & 150.136 & 21.722 & 152.054 \\
7 & 5 & 26.177 & 130.885 & 26.384 & 131.920 \\
8 & 1 & 30.795 & 30.795 & 30.911 & 30.911 \\
9 & 3 & 35.287 & 105.861 & 35.304 & 105.912 \\
\hline Total & 96 & \multicolumn{5}{c}{} & $1,239.584$ \\
\hline
\end{tabular}

$\mathrm{P}=0.45, \mathrm{P}=0.475, \mathrm{~W}=1218.952, \mathrm{~W}=1239.584$.

After linear interpolation, $\mathrm{P}=0.4580, \mathrm{~W}=1225.544, \mathrm{Vp}=\frac{1}{\mathrm{~W}}=0.000816, \sqrt{\mathrm{Vp}}=0.0286$.

$\therefore 45.80 \pm 2.86 \%$

Jpn. J. Human Genet. 
least 2 affected sibs in order to exclude so-called sporadic cases, i.e., $\mathrm{r}_{\min }=2,67$, sibship groups with 297 constituents including 181 affected (100 males and 81 females) were chosen out (Table 2). The calculation of the segregation ratio depended on the population shown in Table 4. If a single selection $(\mathrm{k}=0)$ was applied, the corrected value was;

$$
P=100 \frac{181-67}{297-67}=49.57+3.33 \%
$$

according to the expression (3). The expression (4) gave $59.25 \pm 3.04 \%$ in a complete selection (Table 6). Hence, it was indicated that the segregation ratio in $r_{\min }=2$ lay between $49.57-3.33 \%$ and $59.25 \pm 3.04 \%$.

Table 6. Complete selection (Maximum-likelihood method).

\begin{tabular}{crccccc}
$\mathrm{S}$ & Nsr & Observed & $\frac{\mathrm{SP}}{1-\mathrm{q}^{\mathrm{S}}}$ & $\begin{array}{c}\mathrm{P}=0.575 \\
\text { Expected }\end{array}$ & $\begin{array}{c}\mathrm{SP} \\
1-\mathrm{q}^{\mathrm{s}}\end{array}$ & $\begin{array}{r}\mathrm{P}=0.600 \\
\text { Expected }\end{array}$ \\
\hline 2 & 4 & 18 & 1.404 & 5.616 & 1.429 & 5.716 \\
3 & 17 & 36 & 1.868 & 31.756 & 1.923 & 32.691 \\
4 & 19 & 42 & 2.378 & 45.182 & 2.463 & 46.797 \\
5 & 14 & 43 & 2.915 & 40.810 & 3.031 & 42.434 \\
6 & 6 & 18 & 3.470 & 20.820 & 3.615 & 21.690 \\
7 & 3 & 9 & 4.035 & 12.105 & 4.207 & 12.621 \\
8 & 1 & 4 & 4.605 & 4.605 & 4.803 & 4.803 \\
9 & 3 & 11 & 5.177 & 15.531 & 5.401 & 16.203 \\
\hline Total & 67 & 181 & & 176.425 & & 182.955 \\
\hline
\end{tabular}

$\mathrm{P}=0.575, \mathrm{P}=0.600, \mathrm{R}=176.425, \mathrm{R}=182.955$.

Corrected proportion of affected, after linear interpolation, $P=0.5925(R=181)$.

\begin{tabular}{cccccc}
\hline $\mathrm{S}$ & $\mathrm{Nsr}$ & $\frac{\mathrm{S}\left(1-\mathrm{q}^{\mathrm{s}}-\mathrm{SPq}^{\mathrm{s}-1}\right)}{\mathrm{Pq}\left(1-\mathrm{q}^{\mathrm{s}}\right)^{2}}$ & $\begin{array}{c}\mathrm{P}=0.575 \\
\mathrm{~W}\end{array}$ & $\frac{\mathrm{S}\left(1-\mathrm{q}^{\mathrm{s}}-\mathrm{SPq}^{\mathrm{s}-1}\right)}{\mathrm{Pq}\left(1-\mathrm{q}^{\mathrm{s}}\right)^{2}}$ & $\begin{array}{c}\mathrm{P}=0.600 \\
\mathrm{~W}\end{array}$ \\
\hline 2 & 4 & 4.030 & 16.120 & 4.252 & 17.008 \\
3 & 17 & 8.809 & 149.753 & 9.229 & 156.893 \\
4 & 19 & 13.832 & 262.808 & 14.408 & 273.752 \\
5 & 14 & 18.775 & 262.850 & 19.415 & 271.810 \\
6 & 6 & 23.510 & 141.060 & 24.174 & 145.044 \\
7 & 3 & 28.034 & 84.102 & 28.711 & 86.133 \\
8 & 1 & 32.394 & 32.394 & 33.093 & 33.093 \\
9 & 3 & 36.642 & 109.860 & 37.377 & 112.131 \\
\hline Total & 67 & \multicolumn{5}{c}{} & $1,095.864$ \\
\hline
\end{tabular}

$\mathrm{P}=0.575, \mathrm{P}=0.600, \mathrm{~W}=1058.947, \mathrm{~W}=1095.864$.

After linear interpolation, $\mathrm{P}=0.5925, \mathrm{~W}=1084.789, \mathrm{Vp}=\frac{1}{\mathrm{~W}}=0.000922, \quad \sqrt{\mathrm{Vp}}=0.0304$.

$\therefore 59.25 \pm 3.04 \%$

Vol. 27, No. 4, 1982 
Table 7. Classification of the sibship according to number of affected individuals and size of sibships.

\begin{tabular}{|c|c|c|c|c|c|c|c|c|c|c|c|c|}
\hline & $s=$ & 1 & 2 & 3 & 4 & 5 & 6 & 7 & 8 & 9 & $\sum_{s \geqq 1}(\cdots)$ & $\sum_{s \geqq 2}(\cdots)$ \\
\hline \multirow{5}{*}{$\mathbf{r}=$} & 1 & 2 & 4 & 8 & 2 & 2 & 1 & 2 & & & 21 & 19 \\
\hline & 2 & & 2 & 8 & 8 & 0 & 1 & 1 & & & 20 & 20 \\
\hline & 3 & & & 1 & 1 & 2 & & & & 1 & 5 & 5 \\
\hline & 4 & & & & & 1 & & & & & 1 & 1 \\
\hline & 5 & & & & & & & & & & & \\
\hline \multirow{3}{*}{$r \geqq 1$} & $\sum_{\mathrm{r}} \mathrm{n}_{\mathrm{sr}}$ & 2 & 6 & 16 & 11 & 3 & 5 & 3 & & 1 & 47 & 45 \\
\hline & $\sum_{\mathrm{r}} \mathrm{rn}_{\mathrm{Sr}}$ & 2 & 8 & 24 & 21 & 5 & 13 & 4 & & 3 & 80 & 78 \\
\hline & $\sum_{\mathrm{r} s n_{\mathrm{sr}}}$ & 2 & 12 & 48 & 44 & 15 & 30 & 21 & & 9 & 181 & 179 \\
\hline \multirow{3}{*}{$r \geqq 2$} & $\sum_{r} n_{s r}$ & & 2 & 8 & 9 & 1 & 4 & 1 & & 1 & & 26 \\
\hline & $\sum \mathrm{rrn}_{\mathrm{sr}}$ & & 4 & 16 & 19 & 3 & 12 & 2 & & 3 & & 59 \\
\hline & $\sum_{\mathrm{r}} \mathrm{sn}_{\mathrm{sr}}$ & & 4 & 24 & 36 & 5 & 24 & 7 & & 9 & & 109 \\
\hline
\end{tabular}

The segregation ratios corrected by the penetrance parameter due to delayed onset were calculated on the population shown in Table 7. This population consisted of the sibship groups whose member's ages at the time of this investigation and ages at the onset were all confirmed.

The penetrance parameter due to delayed onset, $y$, is defined as follows.

$$
\begin{aligned}
& y=\int f(z) G(z) d z-\text { complete selection } \\
& y=\int f_{1}(z) G(z) d z-\text { single selection }
\end{aligned}
$$

$f(z)$ : The frequency of the age $z$ at death or last examination among non-affected and affected sibs.

$f_{1}(z)$ : The frequency obtained by excluding index cases from $f(z)$.

$\mathrm{G}(\mathrm{z})$ : The cumulative frequency of the onset age $\mathrm{z}$ among affected sibs.

here, integration covers all the $\mathrm{z}$ range.

By the way, because age $z$ is a discrete probability variable, the penetrance parameter due to delayed onset is defined as follows.

$$
\begin{aligned}
& y=\sum_{z=0}^{x} f(z) G(z)-\text { complete selection } \\
& y=\sum_{z=0}^{x} f_{1}(z) G(z) — \text { single selection }
\end{aligned}
$$

$\mathrm{x}$ : The highest age among non-affected and affected sibs. 
Actual calculation of the population shown in Table 7 , revealed that the penetrance parameters due to delayed onset were 0.67 by a single selection and 0.72 by a complete selection. The result suggested that this poulation consisted of both the sibs suffering from FAP and those not suffering as yet.

The segregation ratios were calculated on the basis of Table 7 according to the expressions (3) and (4). When $r_{\min }=1$, the segregation ratios were $24.63 \pm 3.72 \%$ in applying a single selection $(\mathrm{k}=0)$ and $35.50 \pm 4.36 \%$ in a complete selection $(\mathrm{k}=1)$. When $r_{\min }=2$, the segregation ratios were $39.76 \pm 5.88 \%$ in assuming a single selection $(\mathrm{k}=0)$ and $50.69 \pm 5.34 \%$ in a complete selection $(\mathrm{k}=1)$. The segregation ratios corrected by the penetrance parameters due to delayed onset were $36.76 \%, 49.31 \%$, $59.34 \%$ and $70.40 \%$ respectively (Table 8 ). These values at $r_{\min }=1$ were nearly as large as the foregoing segregation ratios uncorrected and the values at $r_{\min }=2$ were considerably larger than the foregoing values uncorrected.

The uncorrected sex ratio showed a predominance of affected males; that is $1.30: 1$ (120 males and 92 females) when $r_{\min }=1$, and $1.24: 1$ (100 males and 81 females) when $r_{\min }=2$ (Table 9).

Table 8. Segregation ratio and penetrance parameter due to delayed onset in Table 7.

\begin{tabular}{|c|c|c|c|c|c|}
\hline & & \multicolumn{2}{|c|}{ Single selection $(\%)$} & \multicolumn{2}{|c|}{ Complete selection $(\%)$} \\
\hline & & & corrected by c) & & corrected by c) \\
\hline a) & $r_{m I n}=1$ & $24.63 \pm 3.72$ & $* 36.76 \pm 5.55$ & $35.50 \pm 4.36$ & $* 49.31 \pm 6.06$ \\
\hline b) & $r_{\min }=2$ & $39.76 \pm 5.88$ & $* 59.34 \pm 8.78$ & $50.69 \pm 5.34$ & $* 70.40 \pm 7.42$ \\
\hline c) & $\begin{array}{l}\text { Penetrance parameter } \\
\text { due to delayed onset }\end{array}$ & \multicolumn{2}{|c|}{0.67} & \multicolumn{2}{|c|}{0.72} \\
\hline
\end{tabular}

* Corrected by c): a)/c) or b)/c).

Table 9. Assessment of the significance of the sex-ratio.

\begin{tabular}{|c|c|c|c|c|c|c|c|c|c|}
\hline & & \multicolumn{4}{|c|}{ Not corrected } & \multicolumn{4}{|c|}{ Corrected* } \\
\hline & & \multicolumn{3}{|c|}{ Affected sibs } & \multirow[b]{2}{*}{$\chi^{2}$} & \multicolumn{3}{|c|}{ Affected sibs } & \multirow[b]{2}{*}{$\chi^{2}$} \\
\hline & & $\mathrm{m}$ & f & Total & & $\mathrm{m}$ & f & Total & \\
\hline \multirow{2}{*}{$r_{\min }=1$} & Number observed & 120 & 92 & 212 & 3. 70 & 110 & 83 & 193 & 3.78 \\
\hline & Number expected & 106 & 106 & 212 & n.s. & 96.5 & 96.5 & 193 & n.s. \\
\hline \multirow{2}{*}{$r_{\min }=2$} & Number observed & 100 & 81 & 181 & 1.99 & 90 & 72 & 162 & 2.00 \\
\hline & Number expected & 90.5 & 90.5 & 181 & n.s. & 81 & 81 & 162 & n.s. \\
\hline
\end{tabular}

* Corrected: The correction was done after excluding probands (12 males and 10 females) and in assumption that the diagnostically unconfirmed cases were affected according to the segregation ratio $=0.5$.

n.s. : not significant. $p=0.05, \chi^{2}=3.841($ d.f. $=1)$.

Vol. 27, No. 4, 1982 
On calculating $\chi^{2}$ values of the sex ratio in Table 9, we obtained $\chi^{2}=3.70$ when $\mathrm{r}_{\min }=1$ and $\chi^{2}=1.99$ when $\mathrm{r}_{\min }=2$. This result showed that the deviation from the expected value was not significant, because the $\chi^{2}$ value at $p=0.05$ and $f . d .=1$ was 3.841. After excluding probands ( 4 males and 10 females) and assuming that the unconfirmed ( 4 males and 2 females) were affected according to the segregation ratio of $50 \%$, the deviation of these corrected values from the expected ones was still not significant (Table 9).

The calculations of the cumulative morbid risk ratio were made by the next expressions (Matsunaga, 1979).

The morbid risk ratio in i years is defined as follows.

$$
\mathrm{Pi}=\frac{\mathrm{ri}}{\mathrm{ni}}, \quad \mathrm{Vpi}=\frac{\mathrm{ri}(\mathrm{ni}-\mathrm{ri})}{\mathrm{ni} 3}
$$

ni: Number of the individuals who are i years of age and above.

ri: Number of the affected sibs who have suffered from FAP for i years.

Then cumulative morbid risk ratio is;

$$
\mathrm{P}=\Sigma \mathrm{pi}, \quad \mathrm{Vp}=\Sigma \mathrm{Vpi}
$$

Table 10. Cumulative morbid risk in Table 4.

\begin{tabular}{ccc||ccc}
\hline Age & Pi & Vpi & Age & Pi & Vpi \\
\hline 20 & $4.2017 \times 10^{-3}$ & $1.7580 \times 10^{-5}$ & 40 & $2.7211 \times 10^{-2}$ & $1.8007 \times 10^{-4}$ \\
21 & 0 & 0 & 41 & $2.9851 \times 10^{-2}$ & $2.1612 \times 10^{-4}$ \\
22 & $8.5470 \times 10^{-3}$ & $3.6213 \times 10^{-5}$ & 42 & $1.5385 \times 10^{-2}$ & $1.1652 \times 10^{-4}$ \\
23 & 0 & 0 & 43 & $8.9286 \times 10^{-3}$ & $7.9008 \times 10^{-5}$ \\
24 & 0 & 0 & 44 & $9.4340 \times 10^{-3}$ & $8.8160 \times 10^{-5}$ \\
25 & $8.7362 \times 10^{-3}$ & $3.7805 \times 10^{-5}$ & 45 & $1.0526 \times 10^{-2}$ & $1.0964 \times 10^{-4}$ \\
26 & $3.9648 \times 10^{-2}$ & $8.9253 \times 10^{-5}$ & 46 & 0 & 0 \\
27 & $2.6786 \times 10^{-2}$ & $1.1638 \times 10^{-4}$ & 47 & $1.2048 \times 10^{-2}$ & $1.4341 \times 10^{-4}$ \\
28 & $2.6906 \times 10^{-2}$ & $1.1748 \times 10^{-4}$ & 48 & $1.2500 \times 10^{-2}$ & $1.5430 \times 10^{-4}$ \\
29 & $3.1818 \times 10^{-2}$ & $1.4002 \times 10^{-4}$ & 49 & $1.3333 \times 10^{-2}$ & $1.7407 \times 10^{-4}$ \\
30 & $9.3458 \times 10^{-3}$ & $4.3264 \times 10^{-5}$ & 50 & $1.4706 \times 10^{-2}$ & $2.1308 \times 10^{-4}$ \\
31 & $3.4314 \times 10^{-2}$ & $1.6243 \times 10^{-4}$ & 51 & $5.0000 \times 10^{-2}$ & $7.9167 \times 10^{-4}$ \\
32 & $2.5381 \times 10^{-2}$ & $1.2557 \times 10^{-4}$ & 52 & 0 & 0 \\
33 & $1.5625 \times 10^{-2}$ & $8.0109 \times 10^{-5}$ & 53 & $2.0833 \times 10^{-2}$ & $4.2499 \times 10^{-4}$ \\
34 & $3.7634 \times 10^{-2}$ & $1.9472 \times 10^{-4}$ & 54 & 0 & 0 \\
35 & $3.3333 \times 10^{-2}$ & $1.7901 \times 10^{-4}$ & 55 & $2.8095 \times 10^{-2}$ & $5.5340 \times 10^{-4}$ \\
36 & $2.2599 \times 10^{-2}$ & $1.2479 \times 10^{-4}$ & 62 & $8.0000 \times 10^{-2}$ & $2.9440 \times 10^{-3}$ \\
37 & $1.2195 \times 10^{-2}$ & $7.3458 \times 10^{-5}$ & 65 & $5.2632 \times 10^{-2}$ & $2.6243 \times 10^{-3}$ \\
38 & $1.2270 \times 10^{-2}$ & $7.4583 \times 10^{-5}$ & 68 & $7.6923 \times 10^{-2}$ & $5.4620 \times 10^{-3}$ \\
39 & $2.5806 \times 10^{-2}$ & $1.6220 \times 10^{-4}$ & Total & $\mathrm{P}=0.83755$ & Vp=0.016047 \\
\hline
\end{tabular}


Table 11. Cumulative morbid risk in Table 7.

\begin{tabular}{ccc||ccc}
\hline Age & Pi & Vpi & Age & Pi & Vpi \\
\hline 20 & $5.4054 \times 10^{-3}$ & $2.9060 \times 10^{-5}$ & 38 & $8.1301 \times 10^{-3}$ & $6.5561 \times 10^{-5}$ \\
21 & 0 & 0 & 39 & $8.6957 \times 10^{-3}$ & $7.4957 \times 10^{-5}$ \\
22 & $5.4945 \times 10^{-3}$ & $3.0024 \times 10^{-5}$ & 40 & $3.6697 \times 10^{-2}$ & $3.2432 \times 10^{-4}$ \\
23 & 0 & 0 & 41 & $2.9703 \times 10^{-2}$ & $2.8535 \times 10^{-4}$ \\
24 & 0 & 0 & 42 & 0 & 0 \\
25 & $1.1236 \times 10^{-2}$ & $6.2414 \times 10^{-5}$ & 43 & $1.1905 \times 10^{-2}$ & $1.4004 \times 10^{-4}$ \\
26 & $3.9548 \times 10^{-2}$ & $2.1460 \times 10^{-4}$ & 44 & $1.2500 \times 10^{-2}$ & $1.5430 \times 10^{-4}$ \\
27 & $2.8736 \times 10^{-2}$ & $1.6040 \times 10^{-4}$ & 45 & $1.3514 \times 10^{-2}$ & $1.8015 \times 10^{-4}$ \\
28 & $2.3121 \times 10^{-2}$ & $1.3056 \times 10^{-4}$ & 46 & 0 & 0 \\
29 & $2.3392 \times 10^{-2}$ & $1.3359 \times 10^{-4}$ & 47 & 0 & 0 \\
30 & $1.1976 \times 10^{-2}$ & $7.0854 \times 10^{-5}$ & 48 & $1.6667 \times 10^{-2}$ & $2.7315 \times 10^{-4}$ \\
31 & $4.4304 \times 10^{-2}$ & $2.6798 \times 10^{-4}$ & 49 & 0 & 0 \\
32 & $1.3072 \times 10^{-2}$ & $8.4320 \times 10^{-5}$ & 50 & $2.0408 \times 10^{-2}$ & $4.0799 \times 10^{-4}$ \\
33 & $1.3699 \times 10^{-2}$ & $9.2541 \times 10^{-5}$ & 51 & $9.7561 \times 10^{-2}$ & $2.1474 \times 10^{-3}$ \\
34 & $4.2553 \times 10^{-2}$ & $2.8895 \times 10^{-4}$ & 55 & $3.7037 \times 10^{-2}$ & $1.3209 \times 10^{-3}$ \\
35 & $3.6765 \times 10^{-2}$ & $2.6039 \times 10^{-4}$ & 62 & $6.6667 \times 10^{-2}$ & $4.1481 \times 10^{-3}$ \\
36 & $2.9851 \times 10^{-2}$ & $2.1612 \times 10^{-4}$ & 68 & $1.6667 \times 10^{-1}$ & $2.3148 \times 10^{-2}$ \\
37 & $8.0645 \times 10^{-3}$ & $6.4512 \times 10^{-5}$ & Total & $\mathrm{P}=0.86337$ & $\mathrm{Vp}=0.034777$ \\
\hline
\end{tabular}

The cumulative morbid risk ratio in the population consisting of the members whose ages at the time of the investigation and ages at onset were confirmed among the population of Table 4 was 0.83755 (Table 10). The population in Table 7 consisted of the sibship groups whose members' ages at the time of the investigation and the onset were confirmed, and its cumulative morbid risk ratio was 0.86337 (Table 11).

\section{DISCUSSION}

The ages at the onset of FAP cases originating from the Ogawa Village were concentrated between 25 and 45 years. Attention should be paid, however, to the fact the highest age at the onset is 68 years and that the ages at the onset of 5 cases were higher than 60 years. Antunes et al. (1963), Anderson (1970), and Zalin et al. (1974) also reported the cases whose onset ages were higher than 60 years. This suggested that the range of the ages at the onset of FAP is wider than ever thought.

The penetrance ratio from parents' side was $100 \%$, that from children's side was $99.29 \%$ and no skipping of generations was noticed, except a case in which the determination of "affected" or "non-affected" was impossible. Therefore, the transmission mode of FAP originating from the Ogawa Village area was considered to be a regular inheritance. The penetrance ratios from children's side differed with families and ranged from $28.57 \%$ to $200 \%$. This was considered to be distortion due to the scantiness of the constituents of a family. 
Andrade et al. (1969) reported that the segregation ratios of FAP in Portugal were $21.26 \pm 1.88 \%$ by a single selection and $30.84 \pm 2.26 \%$ by a complete selection. Our segregation ratios were larger than Andrade's values. Meretoja (1973) demonstrated that the segregation ratios in the familial amyloidosis with corneal lattice dystrophy and cranial neuropathy in Finland were $42.3 \pm 3.7 \%$ by a single selection and $54.4 \pm 3.7 \%$ by a complete selection. Meretoja's values were larger than ours.

Because the penetrance parameter due to delayed onset, $y$, on the population (Table 7) which consisted of the sibship groups whose members' ages at the time of the investigation and the onset were confirmed was smaller than 1 , the value in the population of Table 4 is also assumed smaller than 1 . Accordingly, it may be conceivable that though the false segregation ratio is smaller than $50 \%$, the true segregation ratio is near to $50 \%$. This can be also assumed from the fact that the penetrance ratio from children's side was $99.29 \%$. (If half of the children suffer from FAP, this ratio is $100 \%$ ).

The transmission mode of FAP originating from the Ogawa Village area may be concluded to be autosomaly dominant, because either one of the two parents of affected sibs of the pedigree map had FAP, the penetrance ratio was considered about $50 \%$ and the affection was not related to the sex.

The sex ratio was $1.30: 1$ and its value of $\chi^{2}$ was 3.70 . Thus, we concluded that this excess of males was not statistically significant, but the difference between this value and $3.841(\mathrm{p}=0.05$, d.f. $=1)$, was generally considered to be little significant (The acceptable level of significance, $\mathrm{p}$ is 0.05 ). According to Table 3 , no matter whether the affected parents are male or female, their children are affected and the number of the male parents nearly equals to that of the females. Although, there was no difference between the sexes in the transmission of FAP from parents to children, the ages at the onset of FAP were 35.38 years in male and 37.88 years in female. Therefore we conclude that this is one of the causes of the deviation from the sex ratio of $1: 1$. Likewise, Andrade et al. (1969) suggested that the apparent variability of penetrance was not due to a sex-controlled gene and that the higher age of manifestation of the disease in female might contribute to the persistence of the gene despite the selection pressures operating against it.

The foregoing two figures of cumulative morbid risk ratio calculated from populations with and without sporadic cases were as high as 0.8 and above. The highness of the cumulative morbid risk ratio and the penetrance ratio suggested high incidence of this disease, in future too.

Kito et al. (1976) performed HLA-typing on 37 cases, 12 pedigrees of which 20 cases were affected and could not find the significant HLA-haplotype and HLAphenotype. On the other hand, Araki and Kito (1980) appointed out the association of BW 52 phenotype of HLA and FAP originated from Arao City.

Linkage studies in a larger population are needed to determine whether these different groups of FAP are genetically homogeneous or not. It is most important 
for us to elucidate how genes participate in the mechanism of manifestation of this disease, because this will lead us to the radical treatment of this disease.

\section{REFERENCES}

Anderson, R. 1970. Hereditary amyloidosis with polyneuropathy. Acta Med. Scand. 188: 85-94.

Andrade, C. 1952. Peculiar form of peripheral neuropathy, familial atypical generalized amyloidosis with special involvement of the peripheral nerves. Brain 75: 407-427.

Andrade, C., Canijo, M., Klein, D., and Kaelin, A. 1969. The genetic aspect of familial amyloidotic polyneuropathy. Portuguese type of paramyloidosis. Humangenetik. 7: 163-175.

Antunes, L., Rosário, M., Ribeirodo, Barros, F., Silva, P., and Coelho, B. 1963. Etudes sur la paranyloidose Portugaise à forme polynévitique (Type C. Andrade). I. Remarques sur le tableau clinique et résultats de quelques examens complémentaires. Acta Neuropathol. Suppl. No. 2: 12-18.

Araki, S., Mawatari, S., Ohta, M., Nakajima, A., and Kuroiwa, Y. 1968. Polyneuritic amyloidosis in a Japanese family. Arch. Neurol. 18: 593-602.

Araki, S. and Kito, S. 1980. Comparative studies on clinical features of familial amyloidotic polyneuropathy in Kumamoto and Nagano prefectures. Annual report of the Ministry of Health and Welfare Amyloid Research Committee, Japan, pp. 95-99.

Izawa, K., Suzuki, M., Honma, Y., and Tsubaki, T. 1969. Familial primary amyloid neuropathy. Report of a case. Clin. Neurol. (Tokyo) 9: 292 (in Japanese).

Kito, S., Fujimori, N., Yamamoto, M., Itoga, E., and Toyozumi, Y. 1973. A new focus of familial amyloid polyneuropathy. Nippon Rinsho 31: 170-182 (in Japanese).

Kito, S., Kusunose, Y., Itoga, E., Yamamoto, M., and Juji, T. 1976. HLA studies on familial amyloid polyneuropathy. Annual report of the Ministry of Health and Welfare Amyloid Research Committee, Japan, pp. 189-196.

Kito, S., Itoga, E., Kamiya, K., Kishida, T., and Yamamura, Y. 1980. Studies on familial amyloid polyneuropathy in Ogawa Village, Japan. Eur. Neurol. 19: 141-151.

Kondo, K. 1976. Genetic epidemiology: The point of contact of genetics and epidemiology. In Neuroepidemiology. Its Principle and Clinical Applications, Kuroiwa, Y. and Kondo, K., eds., Igaku Shoin Ltd., Tokyo, pp. 55-87.

Matsunaga, E. 1979. Pedigree analysis. In Genetics and Medicine I, Inoue, E., ed., Kyoritsu Shuppan Ltd., Tokyo, pp. 39-62.

Meretoja, J. 1973. Genetic aspects of familial amyloidosis with corneal lattice dystrophy and cranial neuropathy. Clinical Genetics 4: 173-185.

Morton, N.E. 1959. Genetic tests under incomplete ascertainment. Am. J. Hum. Genet. 11: 1-16.

Nakao, K., Togi, H., Furukawa, T., Mozai, T., and Toyokura, Y. 1966. A pedigree of familial amyloid neuropathy. Clin. Neurol. (Tokyo) 6: 369-370 (in Japanese).

Oda, M., Furuta, S., Honma, T., Osada, A., and Kumazawa, S. 1974. A pedigree of primary amyloid polyneuropathy. Diag. \& Treatment (Tokyo) 162: 1223-1230.

Ohkura, K. 1979. Proof of geneity: An introduction to human genetics. Igaku Shoin Ltd., Tokyo, pp. 39-62.

Satoyoshi, E., Kinoshita, M., and Suzuki, K. 1971. Two pedigrees of familial primary amyloidosis with a predominant sign of neuropathy. Metab. Dis. (Tokyo) 8: 606-614.

Takaya, G. et al. 1972. An autopsy case of familial amyloidosis being suspected of subacute-myelooptico-neuropathy (SMON). The 63rd Tokyo Clinicopathological Conference.

Tanaka, K. 1978. The method for human genetic study. Basic Human Genetics. Shokabo, Tokyo, pp. 352-375. 
Tsubura, K., Inoue, N., Murata, K., Tsukagoshi, H., and Watanabe, M. 1973. Familial primary amyloid polyneuropathy. Report of two cases. J. Jpn. Soc. Int. Med. 62: 978.

Yamazaki, A., Yoshizawa, O., Shimano, K., Beppu, H., Yoshida, M., and Tsukagoshi, H. 1969. Familial primary amyloid neuropathy. J. Jpn. Soc. Int. Med. 58: 499-505.

Zalin, A., Darby, A., Vaughan, S., and Raftery, E.B. 1974. Primary neuropathic amyloidosis in three brothers. Br. Med.J. 1: 65-66. 